\title{
Wide-Band Integrated Optical Receiver with Improved Dynamic Range Using a Current Switch at the Input
}

\author{
L. A. D. van den Broeke and A. J. Nieuwkerk
}

\begin{abstract}
The front end of optical transmission systems usually consists of a low-noise wide-band negative-feedback transimpedance or current amplifier. The dynamic range of current amplifiers can be extended considerably by bypassing large input currents directly to the output of the amplifier. It is shown that the required current switch does not deteriorate the sensitivity of the receiver. A complete front end, using an external p-i-n photodiode, is integrated in a $2.5-\mathrm{GHz}$ bipolar technology. The receiver has a dynamic range (DR) of $73 \mathrm{~dB}$ in a bandwidth of $220 \mathrm{MHz}$ and consumes a supply current of $1.5 \mathrm{~mA}$.
\end{abstract}

\section{INTRODUCTION}

$\mathbf{T}$ HE most crucial specifications of a receiver front end for an optical transmission system are its bandwidth and its dynamic range (DR). The lower bound of the DR is the noise produced by the front end. The upper bound of the DR, also called the saturation level, is the maximum signal level that can be handled properly.

Many practical receivers consist of an amplifier, integrated in a standard IC process, and an external (pig-tailed) p-i-n photodiode. As wiring capacitance adds to the capacitance of the $\mathrm{p}-\mathrm{i}-\mathrm{n}$, the total capacitance at the input of the amplifier (further denoted as $C_{P I N}$ ) is relatively large (1-2 pF). Negative-feedback amplifiers are most appropriate because they combine a low input impedance, required to avoid loss of signal current into the source capacitance, with low amplifier noise [3]. The output signal of the amplifier is either a voltage or a current, obtained with the configurations from Fig. 1(a) and (b), respectively. Some examples are found in [1] and [2].

In a well-designed amplifier, the lower bound of the DR is determined only by the noise originating from the first stage of the amplifier [3]. This noise is minimized by choosing a suitable configuration of the first amplifier stage (preferably a common-emitter stage) and optimizing its bias current and geometry. By choosing $R_{f}$ sufficiently large (a value of 5-10 $k \Omega$ is generally appropriate), the contribution of its thermal noise can be neglected. In addition, to suppress noise from successive circuitry, the gain of the amplifier should be large enough.

The upper bound of the DR is the saturation level, setting a maximum to the input current (optical power) that can be handled: as the input current causes a voltage across $R_{f}$, the maximum input current is restricted by $R_{f}$ and the maximum signal voltage that can be handled by the output stage of the amplifier. In current amplifiers, the maximum input current is

Manuscript received December 4, 1992; revised March 2, 1993.

The authors are with the Department of Electrical Engineering, Delft University of Technology, $2628 \mathrm{CD}$ Delft, The Netherlands.

IEEE Log Number 9208994.

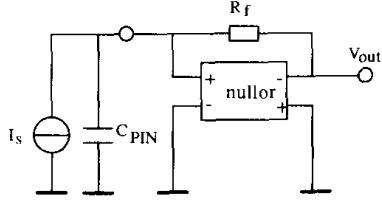

(a)

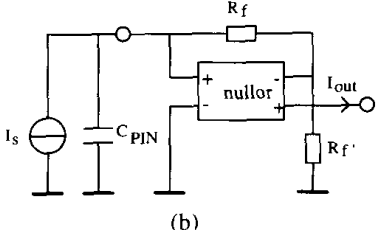

(b)
Fig. 1. Standard configurations of a negative-feedback amplifier having (a) a current-to-voltage and (b) a current-to-current transfer.

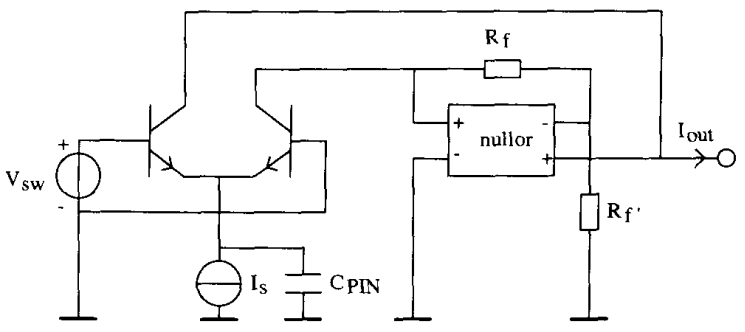

Fig. 2. The current-to-current amplifier with a bypass switch at its input

also restricted by the gain of the amplifier and its maximum output current. Therefore, a high saturation level demands a small value of $R_{f}$ and, in the case of a current amplifier, a low current gain. Consequently, the requirements for a high saturation level conflict with the requirements for a low noise level.

A successful method of enlarging the maximum allowed input signal level is the insertion of a gain control at the input of the amplifier. Unfortunately, continuously variable gain control circuits show poor noise behavior [4]. An alternative solution, suitable for current amplifiers, uses a current switch at the input which bypasses large input signals directly to the output of the amplifier.

We will show that, using the current switch at the amplifier input, the receiver sensitivity is deteriorated only marginally. The realization of a $220-\mathrm{MHz}$ receiver in a $2.5-\mathrm{GHz}$ bipolar process will be discussed and some measurements will be presented.

\section{Configuration of the Current Switch}

The current switch consists of two common-base (CB) stages (Fig. 2). Depending on the voltage $V_{s w}$ across the two bases, the emitter input current is directed to either the input or the output of the amplifier.

An important advantage of this specific type of switch is that it effectively isolates the source capacitance $C_{P I N}$ from the feedback loop of the amplifier. Consequently, unlike that 
in conventional receivers, the high-frequency response of the amplifier does not depend on the actual value of $C_{P I N}$.

The bandwidth of the $\mathrm{CB}$ stage amounts to the transit frequency $f_{t}$ of the transistors. As the maximum bandwidth of negative-feedback amplifiers exhibiting a proper highfrequency behavior is practically restricted to about $f_{t} / 10$, the bandwidth of the switch will be sufficiently large in all cases and needs no further consideration.

\section{NOISE BEHAVIOR}

We will determine the equivalent input noise of $\mathrm{CB}$ stages (adopted in the switch), and that of common-emitter (CE) stages (adopted in the amplifier), when they are connected to capacitive source impedances. Next, we will show that the total noise produced by the switch and successive amplifier is only slightly higher than the noise of an optimally matched amplifier which is connected directly to the p-i-n.

The most dominant noise sources of a bipolar transistor are the collector current shot noise $i_{c}$, the base current shot noise $i_{b}$, and the base bulk resistance thermal noise $u_{b}$. Their power densities are

$$
S\left(i_{c}\right)=2 q I_{c} \quad S\left(i_{b}\right)=2 q \frac{I_{c}}{\beta} \quad S\left(u_{b}\right)=4 k T R_{b}
$$

where $k$ is the Boltzmann constant, $T$ the absolute temperature, $q$ the elementary charge of an electron, $I_{c}$ the collector biasing current, $\beta$ the current gain, and $R_{b}$ the base resistance of the transistor. The sources from (1) are first transformed into equivalent voltage and current noise sources $u_{n}$ and $i_{n}$ in series with the base terminal and in parallel with the base-emitter terminals of the transistor, respectively:

$$
\begin{aligned}
u_{n} & =u_{b}+\frac{k T}{q I_{c}} i_{c} \\
i_{n} & =i_{b}+\left(\frac{1}{\beta}+\frac{j f}{f_{t}}\right) i_{c} \\
f_{t} & =\frac{1}{2 \pi\left(\tau_{f}+\frac{k T}{q I_{c}} C_{j}\right)}
\end{aligned}
$$

where $\tau_{f}$ represents the forward transit time and $C_{j}$ the total base-emitter and base-collector junction capacitance of the transistor.

With $u_{n}$ and $i_{n}$ we are able to compare the noise performance of a $\mathrm{CB}$ with a $\mathrm{CE}$ stage. Fig. 3 shows the transformation of $u_{n}$ and $i_{n}$ to the input and output terminals of the $\mathrm{CB}$ stage. $C_{s}$ is the total source capacitance. The contributions of the resulting voltage sources at the collector terminal are negligible because of the large voltage gain and transimpedance of the CB stage. Transformation of $u_{n}$ and $i_{n}$ in the case of a CE stage, as depicted in Fig. 4, reveals exactly the same equivalent sources at the input. Obviously, CB stages produce the same equivalent input noise as CE stages.

To calculate the total equivalent noise power at the input of a $\mathrm{CB}$ or CE stage, $u_{n}$ and $i_{n}$ are transformed into one equivalent noise current in parallel with $C_{s}$. Integrating its power density spectrum over the amplifier bandwidth $B_{a}$ demonstrates how

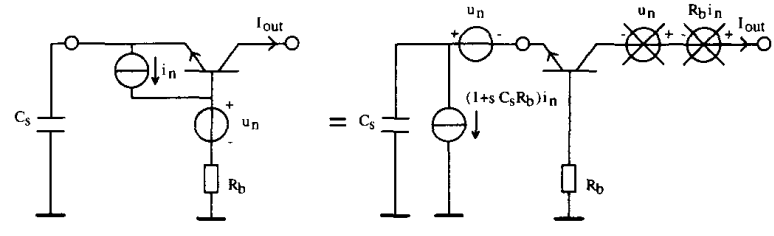

Fig. 3. Determination of the equivalent input noise from $u_{n}$ and $i_{n}$ of the CB stage.

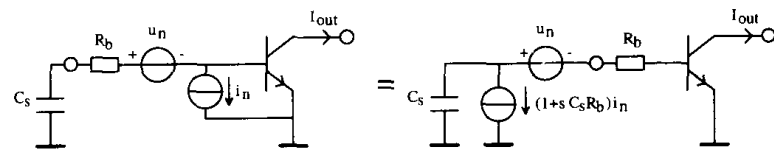

Fig. 4. Determination of the equivalent input noise from $u_{n}$ and $i_{n}$ of a CE stage.

the total noise power depends on $C_{s}$ :

$$
I_{n}^{2} \approx \frac{8 \pi}{3} k T B_{a}^{2}\left(\left(C_{j}+C_{s}\right) \sqrt{\frac{3}{\beta}}+2 \pi B_{a} R_{b} C_{s}^{2}\right) .
$$

In deriving (4) the biasing dependent variable $I_{c}$ was optimized while it was assumed that $B_{a}<\left(1 / 2 \pi \tau_{f}\right) \sqrt{3 / \beta}$. In the case of the switch, $C_{s}$ consists of the total capacitance of the p-i-n photodiode and its wiring $C_{P I N}$, whereas in the case of the successive amplifier, $C_{s}$ consists of the output capacitance of the switch $C_{o}$.

As the switch has unity current gain, the amplifier noise adds to the noise produced by the switch. But, since $C_{o}$, consisting of the relatively small collector-to-substrate and collector-tobase capacitances of the CB stage, is small compared to $C_{P I N}$, the noise contributed by the amplifier may be disregarded. Thus, the noise behavior of the front end is dominated by the switch. Since its equivalent noise equals that of an amplifier using a CE stage at its input, we may conclude that the noise performance of the receiver is not deteriorated by inserting the switch.

So far we have considered asymmetric configurations. When using a balanced input stage, the power density of $i_{n}$ as depicted in Figs. 3 and 4 is halved while the power density of $u_{n}$ is doubled. By taking $R_{b \text {, balanced }}=2 R_{b}$ and $C_{j \text {,balanced }}=C_{j} / 2,(4)$ is approximately correct for the balanced configuration as well.

To avoid clipping, the dc collector current of the CB stage should be larger than the maximum signal current, so optimum biasing is not always possible. However, since for practical implementations the total noise power $I_{n}^{2}$ is not very sensitive to variation of $I_{c}$, this is of minor concern.

\section{Design OF THE RECEIVER}

Fig. 5 shows a balanced configuration of the receiver employing the current switches at the input. The switches consist of $Q_{8}, Q_{9}$ (activated when using the amplifier) and $Q_{7}, Q_{10}$ (activated in the bypass mode). The actual amplifier consists of $Q_{1}-Q_{6}$. The feedback network is constituted around $R_{1}-R_{3}$. Frequency compensation is provided by the base-emitter and base-collector junction capacitances of $Q_{11}, Q_{12}$ and $Q_{13}, Q_{14}$. The high-frequency response of the 


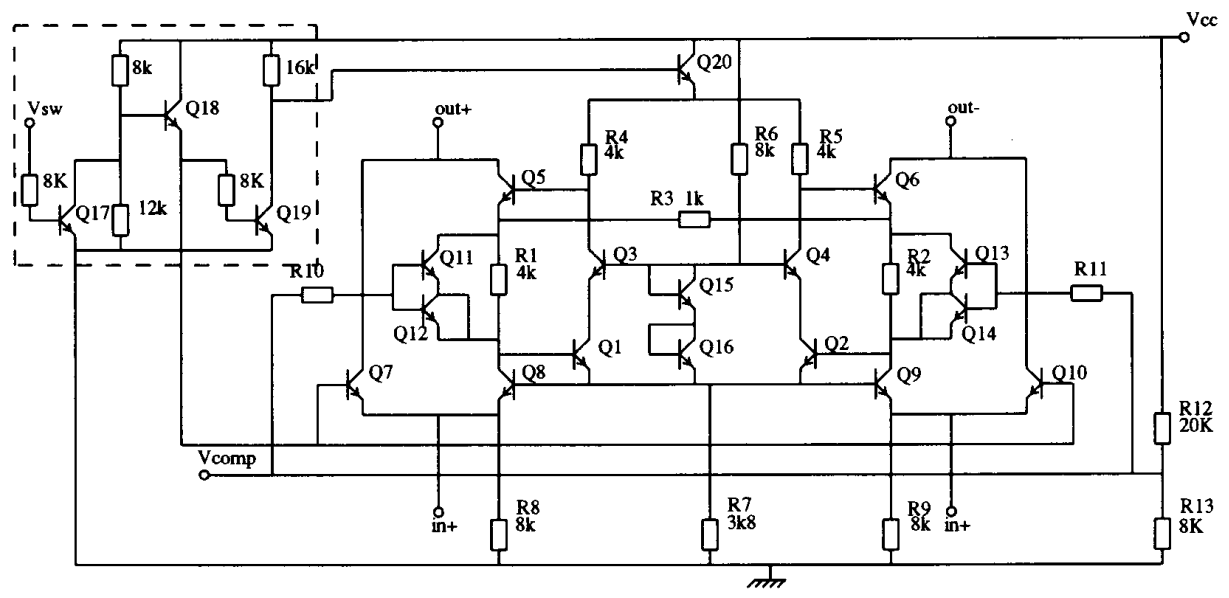

Fig. 5. Circuit diagram of the balanced current amplifier with current switches at the input.

amplifier can be tuned by adjusting the reverse biasing voltages across these junctions.

DC biasing of the current switch and the amplifier is performed by $Q_{15}, Q_{16}$ and $R_{4}-R_{9}$. The circuit within the dashed box drives the current switches and blocks the output stage of the amplifier in the bypass mode. An additional circuit is required for detecting the amplitude of the output signal, deciding the state of the switches. A simple circuit is appropriate to implement this function.

\section{RESULTS}

We have integrated the receiver, which has a bandwidth of $220 \mathrm{MHz}$, in a $2.5-\mathrm{GHz}$ bipolar semicustom process. By substituting $C_{j \text {,balanced }}=0.1 \mathrm{pF}, C_{s}=2 \mathrm{pF}, \beta=100$, and $R_{b \text {, balanced }}=300 \Omega$ into (4), the equivalent input noise current was calculated to be in the order of $60 \mathrm{nA}$.

Fig. 6 depicts the measured noise spectrum at the amplifier output. We have calibrated the $y$ axis in terms of equivalent input noise by applying a reference-level input signal. Integrating the noise spectrum in a bandwidth of $220 \mathrm{MHz}$ results in a total equivalent input noise current of about $75 \mathrm{nA}$, which is only $2 \mathrm{~dB}$ higher than predicted.

As the maximum peak-to-peak input current amplitude equals $1 \mathrm{~mA}$, the $\mathrm{DR}$ is $73 \mathrm{~dB}$.

\section{Conclusions}

The dynamic range of optical receiver front ends can be extended significantly by using a low-noise current switch at the amplifier input that bypasses large input signals directly to its output. Additionally, the switch isolates the capacitance of the photodiode from the amplifier feedback loop, so that this

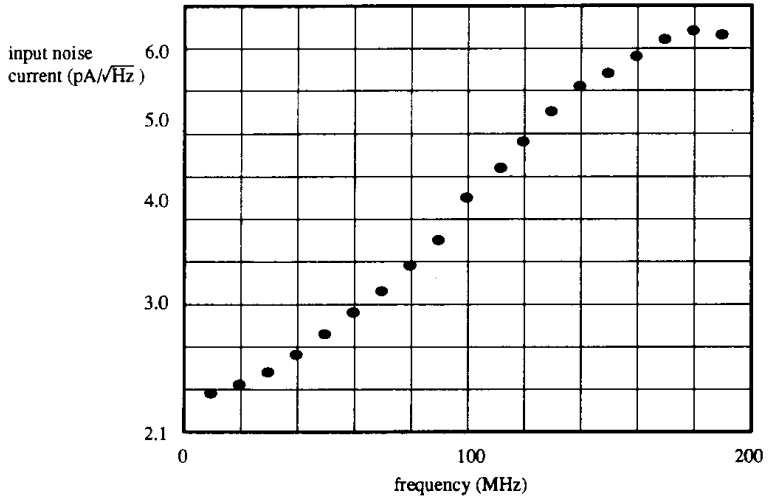

Fig. 6. Measured noise power density spectrum of the receiver.

capacitance has no influence on the amplifier's high-frequency response. A receiver based on this principle was designed and realized in a $2.5-\mathrm{GHz}$ bipolar process. A dynamic range of $73 \mathrm{~dB}$ in a bandwidth of $220 \mathrm{MHz}$ has been achieved.

\section{REFERENCES}

[1] K. Runge et al., "Silicon bipolar integrated circuits for multi-Gb/s optical communication systems," IEEE J. Selected Areas Commun., vol. 9, no, 5 , pp. 636-644, June 1991.

[2] N. Scheinberg, R. J. Bayruns, and T. M. Laverick, "Monolithic GaAs transimpedance amplifiers for fiber-optic receivers," IEEE J. Solid-State Circuits, vol. 26, no. 12, pp. 1834-1839, Dec. 1991.

[3] E. H. Nordholt, Design of High-Performance Negative-Feedback Amplifiers. Amsterdam: Elsevier Scientific, 1983.

[4] R. G. Meyer and W. D. Mack, "A DC to 1-GHz differential monolithic variable-gain amplifier," IEEE J. Solid-State Circuits, vol. 26, no. 11, pp. 1673-1679, Nov. 1991. 